\title{
Fourier Series of the Periodic Bernoulli and Euler Functions
}

\author{
Cheon Seoung Ryoo, ${ }^{1}$ Hyuck In Kwon, ${ }^{2}$ Jihee Yoon, ${ }^{2}$ and Yu Seon Jang ${ }^{3}$ \\ ${ }^{1}$ Department of Mathematics, Hannam University, Daejeon 306-791, Republic of Korea \\ ${ }^{2}$ Department of Mathematics, Kwangwoon University, Seoul 139-701, Republic of Korea \\ ${ }^{3}$ Department of Applied Mathematics, Kangnam University, Yongin 446-702, Republic of Korea
}

Correspondence should be addressed to Yu Seon Jang; ysjang@kangnam.ac.kr

Received 16 April 2014; Revised 16 July 2014; Accepted 16 July 2014; Published 5 August 2014

Academic Editor: Ming Mei

Copyright (C) 2014 Cheon Seoung Ryoo et al. This is an open access article distributed under the Creative Commons Attribution License, which permits unrestricted use, distribution, and reproduction in any medium, provided the original work is properly cited.

We give some properties of the periodic Bernoulli functions and study the Fourier series of the periodic Euler functions which are derived periodic functions from the Euler polynomials. And we derive the relations between the periodic Bernoulli functions and those from Euler polynomials by using the Fourier series.

\section{Introduction}

The numbers and polynomials of Bernoulli and Euler are very useful in classical analysis and numerical mathematics. Recently, several authors have studied the identities of Bernoulli and Euler numbers and polynomials [1-12]. The Bernoulli and Euler polynomials, $B_{n}(x), E_{n}(x), n=$ $0,1, \ldots$, are defined, respectively, by the following exponential generating functions:

$$
\begin{aligned}
& \sum_{n=0}^{\infty} B_{n}(x) \frac{t^{n}}{n !}=\frac{t}{e^{t}-1} e^{x t}, \\
& \sum_{n=0}^{\infty} E_{n}(x) \frac{t^{n}}{n !}=\frac{2}{e^{t}+1} e^{x t} .
\end{aligned}
$$

When $x=0$, these values $B_{n}(0)=B_{n}$ and $E_{n}(0)=E_{n}, n=$ $0,1, \ldots$, are called the Bernoulli numbers and Euler numbers, respectively [13]. als by

Euler polynomials are related to the Bernoulli polynomi-

$$
\begin{aligned}
& E_{n-1}(x)=\frac{2}{n}\left\{B_{n}(x)-2^{n} B_{n}\left(\frac{x}{2}\right)\right\}, \\
& E_{n-2}(x)=\frac{4}{n(n-1)} \sum_{k=0}^{n-2}\left(\begin{array}{l}
n \\
k
\end{array}\right)\left\{\left(2^{n-k}-1\right) B_{n-k} B_{k}(x)\right\},
\end{aligned}
$$

where $\left(\begin{array}{l}n \\ k\end{array}\right)=n ! /(n-k) ! k ![3,14]$.
Bernoulli polynomials and the related Bernoulli functions are of basic importance in theoretical numerical analysis. The periodic Bernoulli functions $\widetilde{B}_{n}(x)$ are Bernoulli polynomials evaluated at the fractional part of the argument $x$ as follows:

$$
\widetilde{B}_{n}(x)=B_{n}(\langle x\rangle)
$$

where $\langle x\rangle=x-[x]$ and $[x]$ is the greatest integer less than or equal to $x$ [13]. Periodic Bernoulli functions play an important role in several mathematical results such as the general Euler-McLaurin summation formula $[1,10,15]$. And also it was shown by Golomb et al. that the periodic Bernoulli functions serve to construct periodic polynomials splines on uniform meshes. For uniform meshes Delvos showed that Locher's method of interpolation by translation is applicable to periodic $B$-splines. This yields an easy and stable algorithm for computing periodic polynomial interpolating splines of arbitrary degree on uniform meshes via Fourier transform [15].

A Fourier series is an expansion of a periodic function $f(x)$ in terms of an infinite sum of sines and cosines. Fourier series make use of the orthogonality relationships of the sine and cosine functions. Since these functions form a 
complete orthogonal system over $[-\pi, \pi]$, the Fourier series of a function $f(x)$ is given by

$$
f(x)=\frac{1}{2} a_{0}+\sum_{n=1}^{\infty}\left(a_{n} \cos n x+b_{n} \sin n x\right),
$$

where

$$
\begin{gathered}
a_{0}=\frac{1}{\pi} \int_{-\pi}^{\pi} f(x) d x, \\
a_{n}=\frac{1}{\pi} \int_{-\pi}^{\pi} f(x) \cos n x d x, \\
b_{n}=\frac{1}{\pi} \int_{-\pi}^{\pi} f(x) \sin n x d x .
\end{gathered}
$$

The notion of a Fourier series can also be extended to complex coefficients $[16,17]$.

The complex form of the Fourier series can be written by the Euler formula, $e^{i \theta}=\cos \theta+i \sin \theta(i=\sqrt{-1})$, as follows:

$$
f(x)=\sum_{k=-\infty}^{\infty} c_{k} e^{i k x}
$$

where

$$
c_{k}=\frac{1}{2 \pi} \int_{-\pi}^{\pi} f(x) e^{-i k x} d x, \quad k \in \mathbb{Z} .
$$

For a function periodic in $[-L / 2, L / 2]$, these become

$$
f(x)=\sum_{n=-\infty}^{\infty} c_{k} e^{i(2 \pi k x / L)},
$$

where

$$
c_{k}=\frac{1}{L} \int_{-L / 2}^{L / 2} f(x) e^{-i(2 \pi k x / L)} d x .
$$

In this paper, we give some properties of the periodic Bernoulli functions and study the Fourier series of the periodic Euler functions which are derived periodic functions from the Euler polynomials. And we derive the relations between the periodic Bernoulli functions and those from Euler polynomials by using the Fourier series. We indebted this idea to Kim $[6-9,18-20]$.

\section{Periodic Bernoulli and Euler Functions}

The periodic Bernoulli functions can be represented as follows:

$$
B_{n}^{*}(x)=B_{n}(x), \quad 0 \leq x<1, n=0,1, \ldots,
$$

satisfying

$$
B_{n}^{*}(x)=B_{n}^{*}(x+1), \quad x \in \mathbb{R}, n=0,1, \ldots
$$

From the definition of $B_{n}(x)$ we know that for $0 \leq x<1$

$$
\sum_{k=0}^{n}\left(\begin{array}{l}
n \\
k
\end{array}\right) B_{k}^{*}(x)-B_{n}^{*}(x)=n x^{n-1}, \quad n=0,1, \ldots
$$

These can be rewritten as follows:

$$
\left(B^{*}(x)+1\right)^{n}-B_{n}^{*}(x)=n x^{n-1}, \quad n=0,1, \ldots,
$$

by using the symbolic convention exhibited by $\left(B^{*}(x)\right)^{n}=$ : $B_{n}^{*}(x)[7]$.

Observe that for $0 \leq x<1$

$$
\sum_{n=0}^{\infty} B_{n}^{*}(1-x) \frac{t^{n}}{n !}=\frac{-t}{e^{-t}-1} e^{x(-t)}=\sum_{n=0}^{\infty}(-1)^{n} B_{n}^{*}(x) \frac{t^{n}}{n !} .
$$

Since $B_{n}^{*}(x), n=0,1, \ldots$, are periodic with period 1 on $\mathbb{R}$, we have

$$
B_{n}^{*}(-x)=(-1)^{n} B_{n}^{*}(x), \quad x \in \mathbb{R} .
$$

The Apostol-Bernoulli and Apostol-Euler polynomials have been investigated by many researchers $[1,2,10,11]$. In [1], Bayad found the Fourier expansion for Apostol-Bernoulli polynomials which are complex version of the classical Bernoulli polynomials. As a result of ordinary Bernoulli polynomials, we have the following lemma.

Lemma 1 (Bayad [1]; see also Luo [10]). The Fourier series of $B_{n}^{*}(x)$ on $(-1,1)$ is

$$
B_{n}^{*}(x)=-n ! \sum_{k \in \mathbb{Z}-\{0\}} \frac{1}{(2 \pi i k)^{n}} e^{2 \pi i k x}, \quad n=0,1, \ldots
$$

From Lemma 1 we have the following theorem.

Theorem 2. For $|x|<1$ and $L \in \mathbb{N}$ one has

$$
\begin{aligned}
& \sum_{\ell=0}^{L-1} B_{n}^{*}\left(\frac{x+\ell}{L}\right) \\
& \quad=\frac{1}{L} B_{n}^{*}\left(\frac{x}{L}\right)-n ! \sum_{k \in \mathbb{Z}-\{0\}}\left[\sum_{\substack{\ell=0 \\
k \neq 0(\bmod L)}}^{L-1} \frac{e^{2 \pi i k(x+\ell) / L}}{(2 \pi i k)^{n}}\right] .
\end{aligned}
$$

Proof. Since

$$
\begin{aligned}
\sum_{\ell=0}^{L-1} B_{n}^{*}\left(\frac{x+\ell}{L}\right)=(-n !) \sum_{\ell=0}^{L-1}\left\{\sum_{k \in \mathbb{Z}-\{0\}} \frac{e^{2 \pi i k(x+\ell) / L}}{(2 \pi i k)^{n}}\right\} \\
=(-n !) \sum_{k \in \mathbb{Z}-\{0\}}\left[\sum_{\substack{\ell=0 \\
k \equiv 0(\bmod L)}}^{L-1} \frac{e^{2 \pi i k(x+\ell) / L}}{(2 \pi i k)^{n}}\right. \\
\left.+\sum_{\substack{\ell=0 \\
k \neq 0(\bmod L)}}^{L-1} \frac{e^{2 \pi i k(x+\ell) / L}}{(2 \pi i k)^{n}}\right]
\end{aligned}
$$

and under $k \equiv 0(\bmod L)$

$$
\sum_{\ell=0}^{L-1} e^{2 \pi i \ell k / L}=L,
$$


we have

$$
\begin{aligned}
& \sum_{\ell=0}^{L-1} B_{n}^{*}\left(\frac{x+\ell}{L}\right) \\
& \quad=(-n !) \sum_{k \in \mathbb{Z}-\{0\}}\left[\frac{1}{L} \frac{e^{2 \pi i k x / L}}{(2 \pi i k)^{n}}+\sum_{\substack{\ell=0 \\
k \neq 0(\bmod L)}}^{L-1} \frac{e^{2 \pi i k(x+\ell) / L}}{(2 \pi i k)^{n}}\right] .
\end{aligned}
$$

This implies the desired result.

The periodic Euler polynomials $\widetilde{E}_{n}(x)$ can be usually defined by $\widetilde{E}_{n}(x+1)=-\widetilde{E}_{n}(x)$ and $\widetilde{E}_{n}(x)=E_{n}(x)$ for $0 \leq$ $x<1$ [13]. Unlike the periodic Bernoulli, which have period 1 , the $\widetilde{E}_{n}(x)$ have periodic 2 and exhibit an even (versus odd) symmetry about zero [21].

As the above Bernoulli case, we consider the periodic Euler functions as the following:

$$
E_{n}^{*}(x)=E_{n}(x), \quad 0 \leq x<1, n=0,1, \ldots,
$$

such that

$$
E_{n}^{*}(x)=E_{n}^{*}(x+1), \quad x \in \mathbb{R}, n=0,1, \ldots
$$

Then the functions $E_{n}^{*}, n=0,1, \ldots$, are also periodic. From definition of Euler polynomials, we know that

$$
\sum_{k=0}^{n}\left(\begin{array}{l}
n \\
k
\end{array}\right) E_{k}^{*}(x)+E_{n}^{*}(x)=2 x^{n}, \quad 0 \leq x<1, n=0,1, \ldots,
$$

where $E_{k}^{*}=E_{k}(0)$ is the $k$ th Euler number. These can be rewritten as follows:

$$
\left(E^{*}(x)+1\right)^{n}+E_{n}^{*}(x)=2 x^{n}, \quad 0 \leq x<1, n=0,1, \ldots,
$$

by using the symbolic convention exhibited by $\left(E^{*}(x)\right)^{n}=$ : $E_{n}^{*}(x)$. When $x=0$, these relations are given by

$$
\left(E^{*}+1\right)^{n}+E_{n}^{*}=2 \delta_{0, n}, \quad n=0,1, \ldots
$$

where $\delta_{0, n}$ is Kronecker symbol and $\left(E^{*}+1\right)^{n}$ is interpreted as $\sum_{k=0}^{n}\left(\begin{array}{c}n \\ k\end{array}\right) E_{k}^{*}[9]$.

Remark 3. Observe that for $0 \leq x<1$

$$
\sum_{n=0}^{\infty} E_{n}^{*}(1-x) \frac{t^{n}}{n !}=\sum_{n=0}^{\infty}(-1)^{n} E_{n}^{*}(x) \frac{t^{n}}{n !} .
$$

As in the Bernoulli case, we have the following equation:

$$
E_{n}^{*}(-x)=(-1)^{n} E_{n}^{*}(x), \quad x \in \mathbb{R} .
$$

This means that if $n$ is odd (even) number, then $E_{n}^{*}$ is odd (even) function.
Theorem 4. For $|x|<1 / 2$, one has

$$
\begin{aligned}
& E_{n}^{*}(x)=-\frac{2}{n+1} E_{n+1}^{*} \\
&+\sum_{k \in \mathbb{Z}-\{0\}}\left[2 \sum_{\ell=1}^{n-2} \frac{(n)_{\ell} E_{n-\ell+1}^{*}}{(2 \pi i k)^{\ell}(k-\ell+1)}\right. \\
&\left.\quad-\frac{n !}{(2 \pi i k)^{n}}\right] e^{2 \pi i k x} .
\end{aligned}
$$

Proof. Let

$$
E_{n}^{*}(x)=\sum_{k=-\infty}^{\infty} c_{k, n}^{*} e^{2 \pi i k x}
$$

be the Fourier series for $E_{n}^{*}(x), n=0,1, \ldots$, on $(-1 / 2,1 / 2)$. Then

$$
c_{k, n}^{*}=\int_{-1 / 2}^{1 / 2} E_{n}^{*}(x) e^{-2 \pi i k x} d x
$$

From definition of $E_{n}^{*}(x)$, we have

$$
\int_{-1 / 2}^{1 / 2} E_{n}(x)^{*} e^{-2 \pi i k x} d x=\int_{0}^{1} E_{n}(x) e^{-2 \pi i k x} d x .
$$

Since $E_{n+1}(1)=-E_{n+1}$, if $k=0$, then

$$
c_{0, n}^{*}=\frac{1}{n+1}\left[E_{n+1}(1)-E_{n+1}\right]=-\frac{2}{n+1} E_{n+1}=-\frac{2}{n+1} E_{n+1}^{*} .
$$

For $k \in \mathbb{Z}-\{0\}$, so we have the following recurrence relation:

$$
\begin{aligned}
c_{k, n}^{*} & =-\frac{2}{n+1} E_{n+1}+\frac{2 \pi i k}{n+1} \int_{0}^{1} E_{n+1}(x) e^{-2 \pi i k x} d x \\
& =-\frac{2}{n+1} E_{n+1}+\frac{2 \pi i k}{n+1} c_{k, n+1}^{*}, \quad n=0,1, \ldots
\end{aligned}
$$

This implies that

$$
\begin{array}{r}
c_{k, n}^{*}=2 \sum_{\ell=1}^{n-2} \frac{(n)_{\ell} E_{n-\ell+1}}{(2 \pi i k)^{\ell}(n-\ell+1)}+\frac{n !}{(2 \pi i k)^{n-1}} c_{k, 1}^{*}, \\
n=0,1, \ldots,
\end{array}
$$

where $(n)_{\ell}=n(n-1) \cdots(n-\ell+1)$ is falling factorial. Since

$$
c_{k, 1}^{*}=\int_{0}^{1}\left(x-\frac{1}{2}\right) e^{-2 \pi i k x} d x=-\frac{1}{2 \pi i k},
$$

we have

$$
c_{k, n}^{*}=2 \sum_{\ell=1}^{n-2} \frac{(n)_{\ell} E_{n-\ell+1}}{(2 \pi i k)^{\ell}(n-\ell+1)}+\frac{n !}{(2 \pi i k)^{n}}, \quad n=0,1, \ldots
$$

This is completion of the proof. 
From Lemma 1 and Theorem 4 we have the following corollary.

Corollary 5. For $|x|<1 / 2$ one has

$$
\begin{aligned}
B_{n}^{*}(x)= & E_{n}^{*}(x)+\frac{2}{n+1} E_{n+1}^{*} \\
& -2 \sum_{k \in \mathbb{Z}-\{0\}}\left[\sum_{\ell=1}^{n-2} \frac{(n)_{\ell} E_{n-\ell+1}^{*}}{(2 \pi i k)^{l}(n-\ell+1)}\right] e^{2 \pi i k x},
\end{aligned}
$$

where $E_{n-\ell+1}^{*}=E_{n-\ell+1}^{*}(0)$ and $(n)_{\ell}=n(n-1) \cdots(n-\ell+1)$ is falling factorial.

Corollary 6. For $|x|<1 / 2$ one has

$$
B_{n}^{*}(x)=E_{n}^{*}(x)+\frac{2}{n+1} E_{n+1}^{*}+2 \sum_{\ell=1}^{n-2}\left(\begin{array}{l}
n \\
\ell
\end{array}\right) \frac{E_{n-\ell+1}^{*}}{n-\ell+1} B_{\ell}^{*}(x),
$$

where $E_{n-\ell+1}^{*}=E_{n-\ell+1}^{*}(0)$.

Proof. From Lemma 1, we have

$$
\begin{aligned}
\sum_{k \in \mathbb{Z}-\{0\}}\left[\sum_{\ell=1}^{n-2} \frac{(n)_{\ell} E_{n-\ell+1}^{*}}{(2 \pi i k)^{\ell}(n-\ell+1)}\right] e^{2 \pi i k x} \\
=\sum_{\ell=1}^{n-2}\left(\begin{array}{l}
n \\
\ell
\end{array}\right) \frac{E_{n-\ell+1}^{*}}{(n-\ell+1)}\left[\ell ! \sum_{k \in \mathbb{Z}-\{0\}} \frac{e^{2 \pi i k x}}{(2 \pi i k)^{\ell}}\right] \\
=\sum_{\ell=1}^{n-2}\left(\begin{array}{l}
n \\
\ell
\end{array}\right) \frac{E_{n-\ell+1}^{*}}{(n-\ell+1)}\left(-B_{\ell}^{*}(x)\right) .
\end{aligned}
$$

This becomes the desired result.

\section{Conflict of Interests}

The authors declare that there is no conflict of interests regarding the publication of this paper.

\section{Acknowledgments}

The authors would like to thank T. Kim for all the motivation and insightful conversations on this subject. The authors would also like to thank the referee(s) of this paper for the valuable comments and suggestions.

\section{References}

[1] A. Bayad, "Fourier expansions for Apostol-Bernoulli, ApostolEuler and Apostol-Genocchi polynomials," Mathematics of Computation, vol. 80, no. 276, pp. 2219-2221, 2011.

[2] D. Ding and J. Yang, "Some identities related to the ApostolEuler and Apostol-Bernoulli polynomials," Advanced Studies in Contemporary Mathematics, vol. 20, no. 1, pp. 7-21, 2010.

[3] K. W. Hwang, D. V. Dolgy, D. S. Kim, T. Kim, and S. H. Lee, "Some theorems on Bernoulli and Euler numbers," Ars Combinatoria, vol. 109, pp. 285-297, 2013.
[4] J. H. Jeong, J. H. Jin, J. W. Park, and S. H. Rim, "On the twisted weak $q$-Euler numbers and polynomials with weight 0 ," Proceedings of the Jangjeon Mathematical Society, vol. 16, no. 2, pp. 157-163, 2013.

[5] D. S. Kim, N. Lee, J. Na, and K. H. Park, "Identities of symmetry for higher-order Euler polynomials in three variables (I)," Advanced Studies in Contemporary Mathematics, vol. 22, no. 1, pp. 51-74, 2012.

[6] T. Kim, "Identities involving Frobenius-Euler polynomials arising from non-linear differential equations," Journal of Number Theory, vol. 132, no. 12, pp. 2854-2865, 2012.

[7] T. Kim, J. Choi, and Y. H. Kim, "A note on the values of Euler zeta functions at posive integers," Advanced Studies in Contemporary Mathematics, vol. 22, no. 1, pp. 27-34, 2012.

[8] T. Kim, D. S. Kim, D. V. Dolgy, and S. H. Rim, "Some identities on the Euler numbers arising from Euler basis polynomials," Ars Combinatoria, vol. 109, pp. 433-446, 2013.

[9] T. Kim, B. Lee, S. H. Lee, and S. H. Rim, "Identities for the Bernoulli and Euler numbers and polynomials," Ars Combinatoria, vol. 107, pp. 325-337, 2012.

[10] Q. Luo, "Fourier expansions and integral representations for the Apostol-Bernoulli and Apostol-Euler polynomials," Mathematics of Computation, vol. 78, no. 268, pp. 2193-2208, 2009.

[11] E. Sen, "Theorems on Apostol-Euler polynomials of higher order arising from Euler basis," Advanced Studies in Contemporary Mathematics, vol. 23, no. 2, pp. 337-345, 2013.

[12] Y. Simsek, O. Yurekli, and V. Kurt, "On interpolation functions of the twisted generalized Frobenius-Euler numbers," Advanced Studies in Contemporary Mathematics, vol. 15, no. 2, pp. 187-194, 2007.

[13] M. Abramowitz and J. Stegun, Handbook of Mathematical Functions with Formulas, Graphs, and Mathematical Tables, Dover, New York, NY, USA, 1972.

[14] M. Aigner, Combinatorial Theory, vol. 234 of Grundlehren der Mathematischen Wissenschaften, Springer, Berlin, Germany, 1979.

[15] F. Delvos, "Bernoulli functions and periodic B-splines," Computing, vol. 38, no. 1, pp. 23-31, 1987.

[16] R. Askey and D. T. Haimo, "Similarities between Fourier and power series," The American Mathematical Monthly, vol. 103, no. 4, pp. 297-304, 1996.

[17] G. B. Folland, Fourier analysis and Its applications, Wadsworth \& Brooks Cole Advanced Books \& Software, Pacific Grove, Calif, USA, 1992.

[18] T. Kim, "Euler numbers and polynomials associated with zeta functions," Abstract and Applied Analysis, vol. 2008, Article ID 581582, 11 pages, 2008.

[19] T. Kim, "On Euler-Barnes multiple zeta functions," Russian Journal of Mathematical Physics, vol. 10, no. 3, pp. 261-267, 2003.

[20] T. Kim, "Note on the Euler numbers and polynomials," Advanced Studies in Contemporary Mathematics, vol. 17, no. 2, pp. 131-136, 2008.

[21] J. M. Borwein, N. J. Calkin, and D. Manna, "Euler-Boole summation revisited," The American Mathematical Monthly, vol. 116, no. 5, pp. 387-412, 2009. 


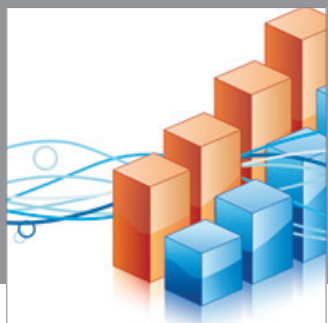

Advances in

Operations Research

mansans



The Scientific World Journal

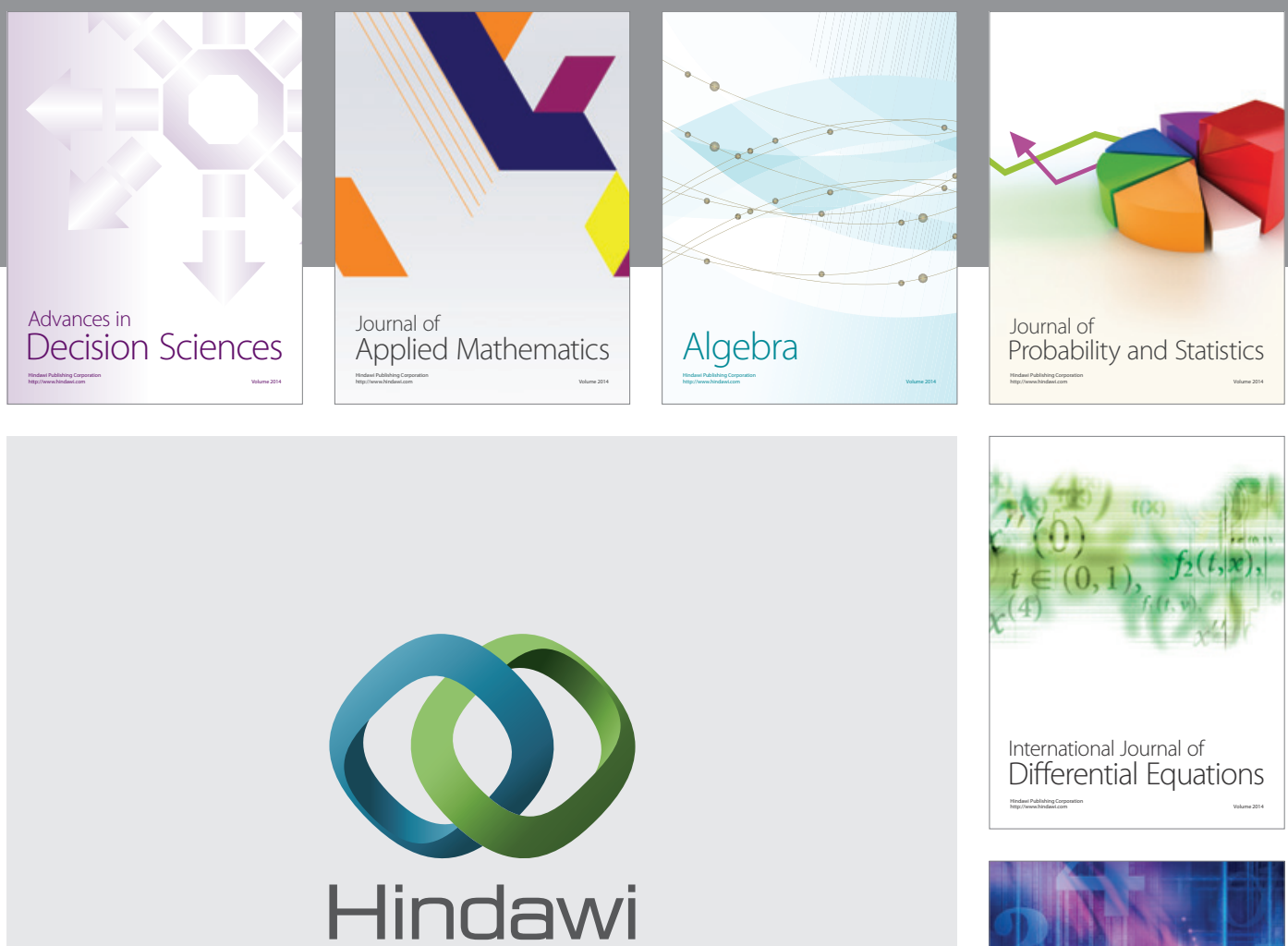

Submit your manuscripts at http://www.hindawi.com
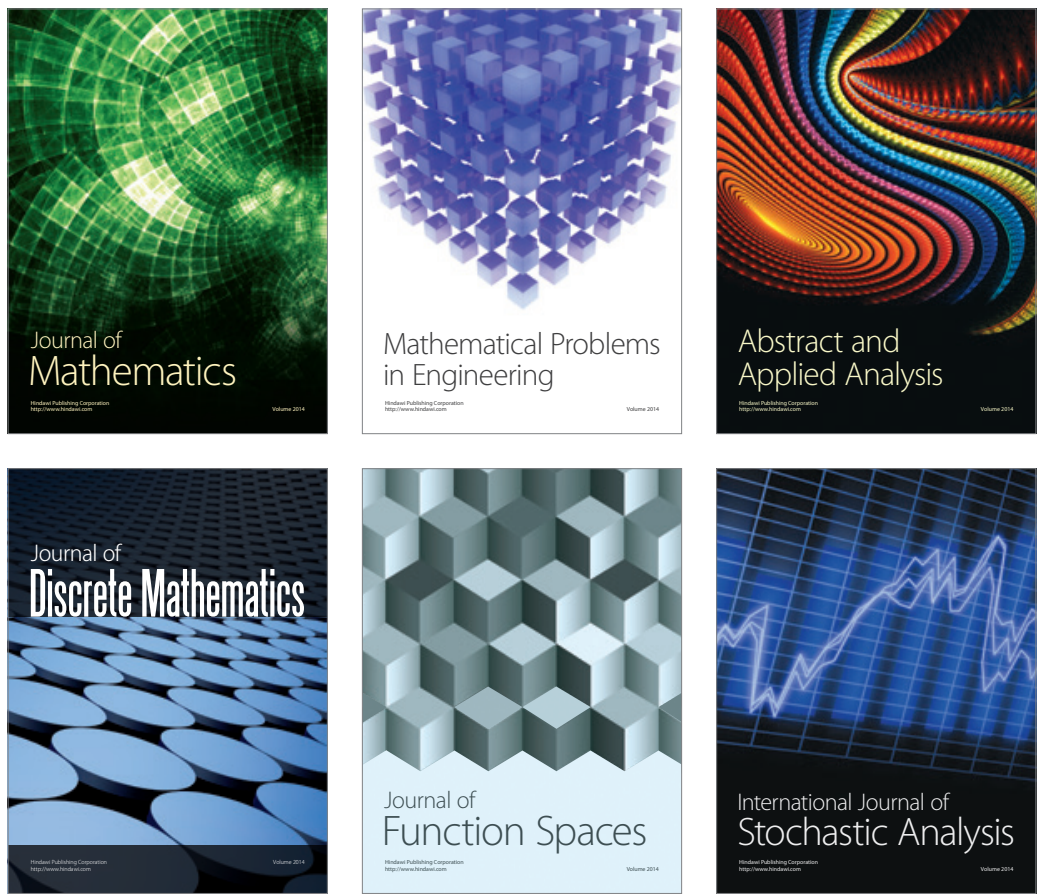

Journal of

Function Spaces

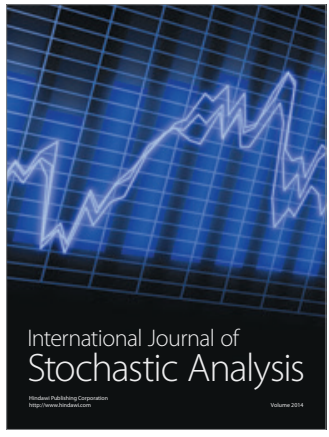


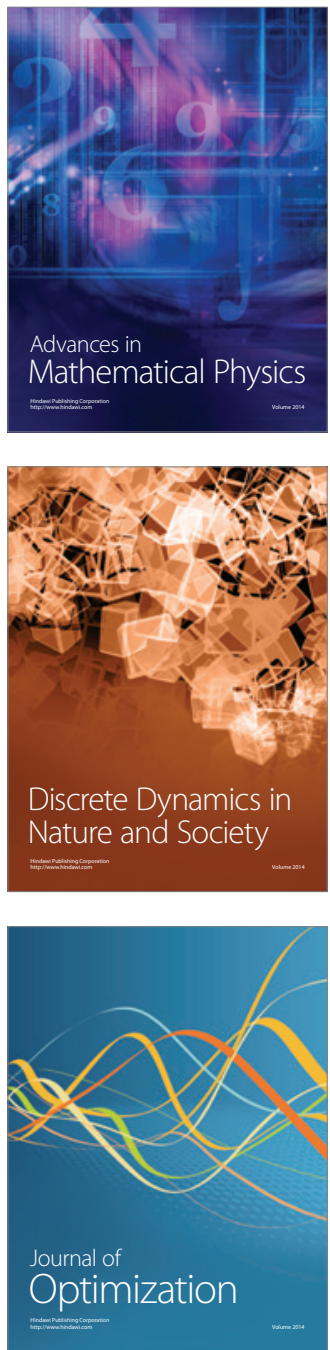